\title{
Development of a low-cost fast-timing microchannel plate photodetector
}

Junqi Xie ${ }^{\mathrm{a}, *}$, Karen Byrum ${ }^{\mathrm{a}, *}$, Marcel Demarteau ${ }^{\mathrm{a}}$, Edward May $^{\mathrm{a}}$, Robert Wagner $^{\mathrm{a}}$, Dean Walters ${ }^{\mathrm{a}}$, Jingbo Wang $^{\mathrm{a}}$, Lei Xia ${ }^{\mathrm{a}}$, Huyue $\mathrm{Zhao}^{\mathrm{a}}$

${ }^{a}$ Argonne National Laboratory, 9700 S Cass Ave., Argonne, IL 60439, USA

\begin{abstract}
We report on the design, fabrication and characterization of a prototype $6 \times 6 \mathrm{~cm}^{2}$ microchannel plate photodetector with precise fast-timing measurement capability. The whole assembly is made of low cost glass materials with a bialkali photocathode top window. All components are hermetically sealed in vacuum. The prototype photodetector exhibits time resolution of 65 ps and $16 \mathrm{ps}$ at single-photoelectron and multi-photoelectron levels, respectively. The spatial resolution reaches $0.54 \mathrm{~mm}$ for multiphotoelectron measurements. The bialkali photocathode exhibits a maximum quantum efficiency exceeding $20 \%$ with a uniformity of $\pm 40 \%$.
\end{abstract}

Keywords: Microchannel plate, MCP, Photodetector, Time resolution, Spatial resolution, Quantum efficiency PACS: $29.40 . \mathrm{Gx}$

\section{Introduction}

The microchannel plate (MCP) photodetector is an evolution from the basic principles of a photomultiplier tube (PMT), by replacing the conventional discrete dynodes with functional MCPs [1]. The pair of thin MCPs enables excellent timing characteristics at the picosecond (1 ps $\left.=10^{-12} \mathrm{~s}\right)$ level [2]. Good spatial resolution at the micrometer $\left(1 \mu \mathrm{m}=10^{-6} \mathrm{~m}\right)$ level [3] is also obtained with MCP-based detectors through various readout configurations. These precise timing and position measurements make MCP photodetectors an attractive candidate for applications in various fields, such as high precision time-of-flight (TOF) systems, positron emission tomography (PET) and large Cherenkov detectors if the detectors are cost-competitive.

Recently, the production of a new class of low-cost microchannel plates has been commercialized by Incom, Inc. [4]. The MCP production process applies atomic layer deposition (ALD) [5] on capillary glass channel substrates, producing MCPs with better gain uniformity, much longer lifetime, more robust performance and potentially at a much lower cost than the standard commercial MCPs made of lead glass. This innovation has provided new opportunities for fast timing MCP photodetector development.

At Argonne National Laboratory, we have designed and built a photodetector production facility capable of producing $6 \times 6$ $\mathrm{cm}^{2} \mathrm{MCP}$ photodetectors [6]. The facility incorporates vacuum photocathode growth with MCP scrubbing and vacuum sealing, and serves as a test bed for fast timing photodetector research and development. In this work, we fabricated prototype $6 \times 6$ $\mathrm{cm}^{2} \mathrm{MCP}$ photodetectors with exceptional timing characteris-

\footnotetext{
${ }^{*}$ Corresponding authors:

Email addresses: jxie@anl.gov (Junqi Xie), byrum@anl.gov (Karen Byrum)
}

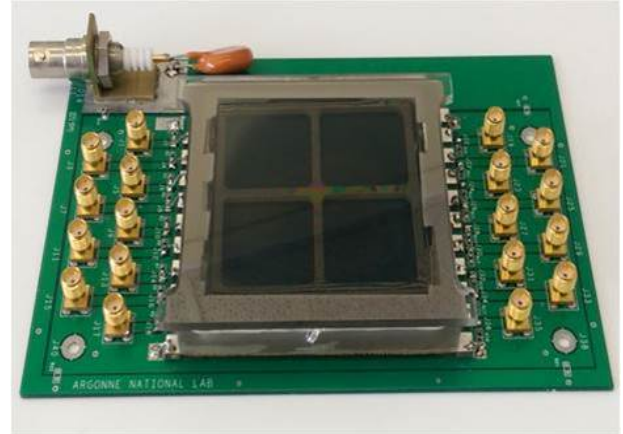

Figure 1: Picture of a prototype $6 \mathrm{~cm} \times 6 \mathrm{~cm}$ MCP photodetector with resistor chain design, assembled completely with borosilicate glass.

tics. The detailed design, fabrication and characterization of the prototype MCP photodetectors are presented.

\section{Design and fabrication of the MCP photodetector}

As a proof of concept, the $6 \times 6 \mathrm{~cm}^{2} \mathrm{MCP}$ photodetector design was adapted from the pin-less resistor chain design [7]. The whole assembly envelope is composed of low-cost borosilicate float glass. Two MCPs and three ALD coated spacers are stacked together, forming a continuous resistive path between cathode and anode. During photodetector operation, a negative high voltage is applied on the top photocathode glass window, and the strip-line anode is connected to the DC ground. High voltages across the MCPs and spacers are then determined by their resistances. Normally, a voltage drop of $\sim 1000 \mathrm{~V}$ is applied across each MCP.

The fabrication of the $6 \times 6 \mathrm{~cm}^{2} \mathrm{MCP}$ photodetector consists of four major steps: (1) MCP and spacer functionalization; (2) MCP baking and scrubbing; (3) photocathode growth; and (4) 


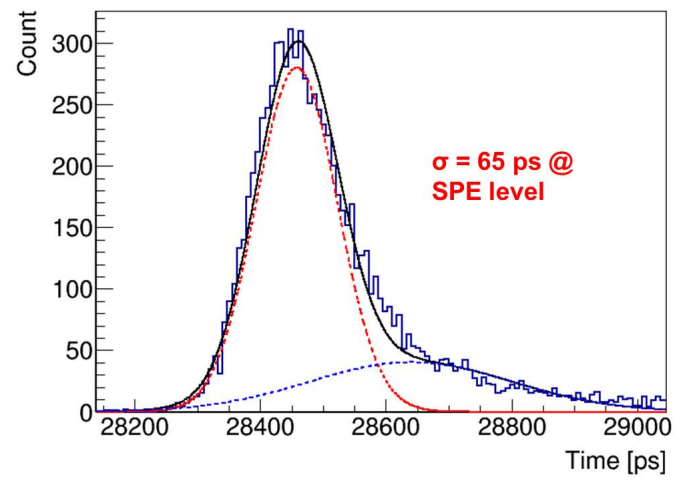

Figure 2: Transit time spread distribution of MCP photodetector at single photoelectron level with time resolution of 65 ps.

thermo-compression sealing. The as-received glass capillary arrays are functionalized with two ALD coatings to make them suitable for use as MCPs in the photodetector. A thin resistive and emissive layer is added, providing the MCPs with appropriate conductivity and high secondary electron yield. The spacers are only coated with the resistive layer for appropriate conductivity. A "scrubbing" process is applied to the MCP pair and spacer stack to remove the gas molecules adsorbed inside the microchannel pores, stabilizing the MCP gain as well as improving the photodetector lifetime. Scrubbing is the process of producing a low gain continuous current of electrons in the MCPs so as to remove physisorbed and chemisorbed contaminants from the MCP pores. We generate the electrons directly using a cold photocathode gun. Scrubbing can also be accomplished using a UV lamp to eject electrons from the ALD surface of the MCPs themselves. The process lowers the longterm outgassing of the MCPs, helping to maintain UHV conditions in the sealed tube. Following the scrubbing process, a bialkali antimonide $\left(\mathrm{K}_{2} \mathrm{CsSb}\right)$ photocathode is deposited on a glass top window and transferred in vacuum to a sealing chamber. The photocathode, the scrubbed MCP-spacer stack and the anode plate are sealed in a vacuum envelope, producing a prototype 6 $\times 6 \mathrm{~cm}^{2} \mathrm{MCP}$ photodetector, as shown in Figure 1 .

\section{Characteristics of the MCP photodetector}

\subsection{Timing characteristics}

Timing characteristics were measured on an in-house detector characterization station, composed of a Hamamatsu PLP laser with $10 \mathrm{ps}$ pulses at $405 \mathrm{~nm}$, beam alignment system, neutral density (ND) filters and a Tektronix DPO $73453.5 \mathrm{GHz}$ bandwidth, $10 \mathrm{Gs} / \mathrm{s}$ (Giga samples per second) digital oscilloscope. Two different light intensities were chosen to evaluate the performance of the prototype MCP photodetector: (1) single photoelectron level (SPE) for applications focused on SPE or near SPE response; and (2) multi-photoelectron level (>120 PEs) for applications interested in high light intensity.

The timing resolution capability of an MCP photodetector can be expressed as the standard deviation of the transit-time

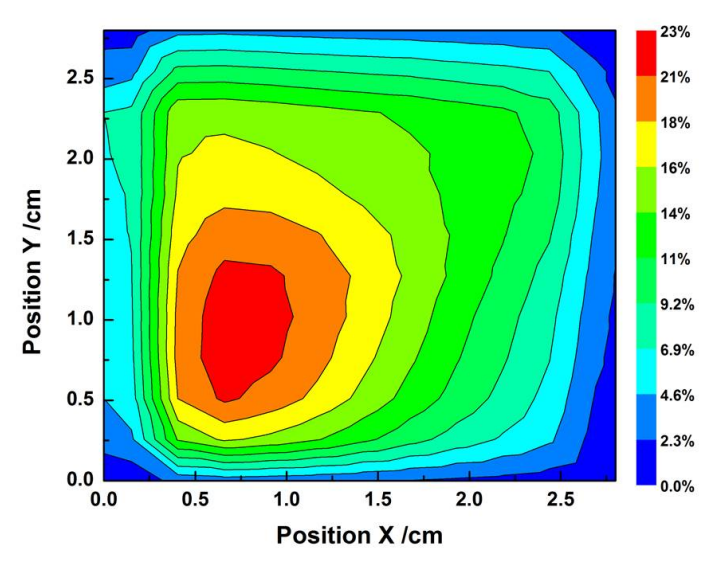

Figure 3: QE map of the $\mathrm{K}_{2} \mathrm{CsSb}$ photocathode with $3 \times 3 \mathrm{~cm}^{2}$ activation area, showing a maximum QE over $20 \%$.

spread (TTS) distribution, i.e. the time interval between the arrival of a light pulse at the photocathode and the appearance of the output pulse. Figure 2 shows the TTS distribution of the prototype MCP photodetector triggered by laser pulses at the single photoelectron level. A Gaussian fit of the TTS distribution gives a standard deviation of $\sigma(\mathrm{t})=65 \mathrm{ps}$ at the single photoelectron level. The timing resolution of the MCP photodetector at the multi-photoelectron level was also measured, giving a standard deviation of $\sigma(\mathrm{t})=16 \mathrm{ps}$.

The spatial resolution is evaluated by determining the differential time resolution (i.e. time difference of a signal arrival at the opposite ends of an anode strip-line). The best differential time resolution we measured was 6 ps at the multiphotoelectron level, corresponding to a spatial resolution of $0.54 \mathrm{~mm}$.

\subsection{Photocathode characteristics}

The photocathode quantum efficiency (QE) was characterized on an in-house optical-elctrical station [8] by measuring the increase in bias current when the photocathode is exposed to light while the detector is biased. Because of the relatively large dark current due to the current resistive chain design, this method has a very small signal to noise ratio and hence a relatively large uncertainty on the measurement of current photocathode QE. In order to measurement the QE accurately, we fabricated a new photodetector with the MCPs biased individually. In this case, the photocathode QE can be measured accurately by applying bias voltage between photocathode and the upper surface of the top MCP. Figure 3 shows part of the $\mathrm{K}_{2} \mathrm{CsSb}$ photocathode QE distribution map since this new photodetector was not sealed sucessfully, other areas of the photocathode were deteriorated. The photocathode with the $3 \times 3$ $\mathrm{cm}^{2}$ active area exhibits a maximum QE exceeding $20 \%$ with a uniformity of $\pm 40 \%$. Further work on improving the QE uniformity is currently ongoing. 


\section{Conclusion}

In conclusion, prototype MCP photodetectors with precise fast-timing measurement capability were fabricated and characterized. The MCP photodetector is composed of a bialkali photocathode, MCP-spacer stack and glass base with a strip-line anode. The prototype photodetector exhibits time resolution of $65 \mathrm{ps}$ and $16 \mathrm{ps}$ at single-photoelectron and multi-photoelectron levels, respectively. The spatial resolution reaches $0.54 \mathrm{~mm}$ for multi-photoelectron measurements. The photocathode exhibits a maximum quantum efficiency over $20 \%$. The future work will be focused on fabricating MCP-photodetectors with a new design of independently biased MCPs and improving the QE uniformity of the bialkali photocathode.

\section{Acknowledgments}

Work at Argonne National Laboratory was supported by the U. S. Department of Energy, Office of Science, Office of Basic Energy Sciences and Office of High Energy Physics under contract DE-AC02-06CH11357.

\section{References}

[1] A. Barnyakov, et al., R\&D of microchannel plate phototubes, Nucl. Instrum. Meth. A 567 (2006) 17-20.

[2] J. Milnes and J. Howorth, Picosecond time response of microchannel plate PMT detectors, Proc. SPIE 5580 (2005) 730-740.

[3] A. Tremsin and O. Siegmund, Spatial distribution of electron cloud footprints from microchannel plates: Measurements and modeling, Rev. Sci. Instrum. 70 (1999) 3282-3288.

[4] M. Minot, et al., Pilot production \& commercialization of LAPPD ${ }^{\mathrm{TM}}$, Nucl. Instrum. Meth. A 787 (2015) 78-84.

[5] A. Mane, et al., An atomic layer deposition method to fabricate economical and robust large area microchannel plates for photodetectors, Physics Procedia 37 (2012) 722-732.

[6] J. Xie, et al., Development of a small form factor $(6 \mathrm{~cm} \times 6 \mathrm{~cm})$ picosecond photodetector as a path towards the commercialization of large area devices, in: Proceeding of "The Technology and Instrumentation in Particle Physics 2014", PoS (TIPP2014) (2014) 233.

[7] B. Adams, et al., An internal ALD-based high voltage divider and signal circuit for MCP-based photodetectors, Nucl. Instrum. Meth. A 780 (2015) $107-113$.

[8] J. Xie, et al., Instrumentation for theory-inspired photocathode development within the large area picosecond photodetector (LAPPD) project, Phys. Procedia 37 (2012) 811. 